\title{
Lie Groups Actions on Non Orientable $n$-Dimensional Complex Manifolds
}

\author{
T. Cao-Huu, D. Ghisa \\ Glendon College, York University, Toronto, Canada \\ Email: tuan@gl.yorku.ca,dghisa@yorku.ca
}

How to cite this paper: Cao-Huu, T. and Ghisa, D. (2021) Lie Groups Actions on Non Orientable $n$-Dimensional Complex Manifolds. Advances in Pure Mathematics, 11, 604-610.

https://doi.org/10.4236/apm.2021.116039

Received: May 13, 2021

Accepted: June 27, 2021

Published: June 30, 2021

Copyright $\odot 2021$ by author(s) and Scientific Research Publishing Inc. This work is licensed under the Creative Commons Attribution International License (CC BY 4.0).

http://creativecommons.org/licenses/by/4.0/

\section{(c) (i) Open Access}

\begin{abstract}
Analytic atlases on $\overline{\mathbb{C}}^{n}$ can be easily defined making it an $n$-dimensional complex manifold. Then with the help of bi-Möbius transformations in complex coordinates Abelian groups are constructed making this manifold a Lie group. Actions of Lie groups on differentiable manifolds are well known and serve different purposes. We have introduced in previous works actions of Lie groups on non orientable Klein surfaces. The purpose of this work is to extend those studies to non orientable $n$-dimensional complex manifolds. Such manifolds are obtained by factorizing $\overline{\mathbb{C}}^{n}$ with the two elements group of a fixed point free antianalytic involution of $\overline{\mathbb{C}}^{n}$. Involutions $\mathbf{h}(\mathbf{z})$ of this kind are obtained linearly by composing special Möbius transformations of the planes with the mapping $k(z)=-1 / \bar{z}$. A convenient partition of $\overline{\mathbb{C}}^{n}$ is performed which helps defining an internal operation on $\overline{\mathbb{C}}^{n} /\langle\mathbf{h}\rangle$ and finally actions of the previously defined Lie groups on the non orientable manifold $\overline{\mathbb{C}}^{n} /\langle\mathbf{h}\rangle$ are displayed.
\end{abstract}

\section{Keywords}

Analytic Atlas, Complex Manifold, Möbius Transformation, Lie Group Action

\section{Introduction}

We dealt in [1] and [2] with Lie groups of bi-Möbius transformations defined on $\overline{\mathbb{C}}$. The concept can be extended linearly to $\overline{\mathbb{C}}^{n}$ in the following way.

If $\mathbf{z} \in \overline{\mathbb{C}}^{n}$ then $\mathbf{z}=\left(z_{1}, z_{2}, \cdots, z_{n}\right), z_{k} \in \overline{\mathbb{C}}, k=1,2, \cdots, n$. Let us study the function f: $: \overline{\mathbb{C}}^{n} \times \overline{\mathbb{C}}^{n} \rightarrow \overline{\mathbb{C}}^{n}$ defined by

$$
\mathbf{f}(\mathbf{z}, \mathbf{w})=\left(f_{1}\left(z_{1}, w_{1}\right), f_{2}\left(z_{2}, w_{2}\right), \cdots, f_{n}\left(z_{n}, w_{n}\right)\right)
$$


where $f_{k}\left(z_{k}, w_{k}\right)=\left[A_{k} z_{k} w_{k}+a_{k}\left(1-z_{k}-w_{k}\right)\right] /\left[a_{k}\left(z_{k} w_{k}-z_{k}-w_{k}\right)+A_{k}\right]$, $A_{k}=a_{k}^{2}-a_{k}+1, \quad a_{k} \in \overline{\mathbb{C}} \backslash\{0,1\}, f_{k}\left(z_{k}, \infty\right)=\left[\left(a_{k}-1+1 / a_{k}\right) z_{k}-1\right] /\left(z_{k}-1\right)$, $f_{k}\left(\infty, w_{k}\right)=\left[\left(a_{k}-1+1 / a_{k}\right) w_{k}-1\right] /\left(w_{k}-1\right), \quad f_{k}(\infty, \infty)=a_{k}-1+1 / a_{k}$.

Theorem 1. Let us denote $\mathbf{1}=(1,1, \cdots, 1)$ and $\mathbf{a}=\left(a_{1}, a_{2}, \cdots, a_{n}\right) \neq \mathbf{1}$, $\mathbf{z}^{-1}=\left(1 / z_{1}, 1 / z_{2}, \cdots, 1 / z_{n}\right)$. The function $\mathbf{f}(\mathbf{z}, \mathbf{w})$ satisfies the following relations:

1) $\mathbf{f}(\mathbf{z}, \mathbf{w})=\mathbf{f}(\mathbf{w}, \mathbf{z})$, for every $\mathbf{z}, \mathbf{w} \in \overline{\mathbb{C}}^{n}$

2) $\mathbf{f}(\mathbf{z}, \mathbf{1})=\mathbf{z}$ and $\mathbf{f}(\mathbf{1}, \mathbf{w})=\mathbf{w}$, for every $\mathbf{z}, \mathbf{w} \in \overline{\mathbb{C}}^{n}$

3) $\mathbf{f}\left(\mathbf{z}, \mathbf{z}^{-1}\right)=\mathbf{1}$, for every $\mathbf{z} \in \overline{\mathbb{C}}^{n}$

4) $\mathbf{f}\left(\mathbf{z}, \mathbf{w}^{-1}\right)=\mathbf{f}\left(\mathbf{z}^{-1}, \mathbf{w}\right)^{-1}$, hence $\mathbf{f}\left(\mathbf{z}^{-1}, \mathbf{w}^{-1}\right)=\mathbf{f}(\mathbf{z}, \mathbf{w})^{-1}$, for every $\mathbf{z}, \mathbf{w} \in \overline{\mathbb{C}}^{n}$

5) $\mathbf{f}(\mathbf{u}, \mathbf{f}(\mathbf{v}, \mathbf{w}))=\mathbf{f}(\mathbf{f}(\mathbf{u}, \mathbf{v}), \mathbf{w})$ for every $\mathbf{u}, \mathbf{v}, \mathbf{w} \in \overline{\mathbb{C}}^{n}$

6) $\mathbf{f}(\mathbf{z}, \mathbf{a})=\mathbf{f}(\mathbf{a}, \mathbf{w})=\mathbf{a}$ and $\mathbf{f}\left(\mathbf{z}, \mathbf{a}^{-1}\right)=\mathbf{f}\left(\mathbf{a}^{-1}, \mathbf{w}\right)=\mathbf{a}^{-1}$ for every $\mathbf{z}, \mathbf{w}, \mathbf{a} \in \overline{\mathbb{C}}^{n}$. Moreover, $\mathbf{f}(\mathbf{z}, \mathbf{w})=\mathbf{a}$ only if $\mathbf{z}=\mathbf{a}$ or $\mathbf{w}=\mathbf{a}$ and $\mathbf{f}(\mathbf{z}, \mathbf{w})=\mathbf{a}^{-1}$ only if $\mathbf{z}=\mathbf{a}^{-1}$ or $\mathbf{w}=\mathbf{a}^{-1}$.

It results that the composition law $\mathbf{z} \circ \mathbf{w}=\mathbf{f}(\mathbf{z}, \mathbf{w})$ defines a structure of $A b$ elian group on $\overline{\mathbb{C}}^{n}$ with the unit element $\mathbf{1}$ and $\mathbf{z}^{-1}$ the inverse element of z .

Proof. The proof requires only elementary computation. For (5) it is enough to show that $f_{k}\left(u_{k}, f_{k}\left(v_{k}, w_{k}\right)\right)=f_{k}\left(f_{k}\left(u_{k}, v_{k}\right), w_{k}\right)$ for arbitrary $k=1,2, \cdots, n$ and this results again after an elementary (although a little more tedious) computation. The relation (6) shows that by removing the elements $\mathbf{z}=\mathbf{a}$ and $\mathbf{z}=\mathbf{a}^{-1}$ we obtain a subgroup $\mathbf{G}_{\mathbf{a}}$ of this group. The functions

$$
g_{z_{k}}\left(w_{k}\right)=\frac{\left(A_{k} z_{k}-a_{k}\right) w_{k}-a_{k}\left(z_{k}-1\right)}{a_{k}\left(z_{k}-1\right) w_{k}+A_{k}-a_{k} z_{k}}, \quad z_{k} \in \overline{\mathbb{C}} \backslash\left\{a_{k}, 1 / a_{k}\right\}
$$

are Möbius transformations, since $\left(A_{k} z_{k}-a_{k}\right)\left(A_{k}-a_{k} z_{k}\right)+a_{k}^{2}\left(z_{k}-1\right)^{2} \neq 0$ as long as $z_{k} \neq a_{k}$ and $z_{k} \neq 1 / a_{k}$, which has been postulated. Moreover, due to the fact that $g_{z_{k}}$ are injective and taking into account Theorem 1 (6), $g_{z_{k}}\left(w_{k}\right)=a_{k}$ if and only if $w_{k}=a_{k}$ and $g_{z_{k}}\left(w_{k}\right)=1 / a_{k}$ if and only if $w_{k}=1 / a_{k}$. These Möbius transformations induce transformations of $\overline{\mathbb{C}}^{n}$ defined by

$$
\mathbf{g}_{\mathbf{z}}(\mathbf{w})=\left(g_{z_{1}}\left(w_{1}\right), g_{z_{2}}\left(w_{2}\right), \cdots, g_{z_{n}}\left(w_{n}\right)\right)
$$

Theorem 2. The set of transformations $\mathbf{G}=\left\{\mathbf{g}_{\mathbf{z}} \mid \mathbf{z} \in \overline{\mathbb{C}}^{n} \backslash\left\{\mathbf{a}, \mathbf{a}^{-1}\right\}\right\}$ endowed with the composition law

$$
\mathbf{g}_{\mathbf{z}} \times \mathbf{g}_{\zeta}=\mathbf{g}_{\mathbf{z} \circ \zeta}
$$

is an Abelian group having the identity element $\mathbf{g}_{1}$ and such that the inverse element of $\mathbf{g}_{\mathbf{z}}$ is $\mathbf{g}_{\mathbf{z}^{-1}}$.

This group is isomorphic with $\mathbf{G}_{\mathbf{a}}$, the isomorphism being given by the mapping $\chi\left(\mathbf{g}_{\mathbf{z}}\right)=\mathbf{z}$. It makes $\mathbf{G}$ a Lie group with analytic structure as $n$-dimensional complex differentiable manifold.

Proof. Indeed, if $\mathbf{z}, \zeta \in \overline{\mathbb{C}}^{n} \backslash\left\{\mathbf{a}, \mathbf{a}^{-1}\right\}$, then by Theorem 1 (6) $\mathbf{z} \circ \zeta \in \overline{\mathbb{C}}^{n} \backslash\left\{\mathbf{a}, \mathbf{a}^{-1}\right\}$, 
hence $\mathbf{g}_{\mathrm{z}} \times \mathbf{g}_{\zeta}=\mathbf{g}_{\mathrm{z} \circ \zeta} \in \mathbf{G}$.

The commutativity results from: $\mathbf{g}_{\mathbf{z}} \times \mathbf{g}_{\zeta}=\mathbf{g}_{\mathbf{z} \circ \zeta}=\mathbf{g}_{\zeta \circ \mathbf{z}}=\mathbf{g}_{\zeta} \times \mathbf{g}_{\mathbf{z}}$.

The identity element is $\mathbf{g}_{1}$, since $\mathbf{g}_{\mathbf{z}} \times \mathbf{g}_{1}=\mathbf{g}_{\mathbf{z} 01}=\mathbf{g}_{\mathbf{z}}$.

The composition law is associative since:

$\mathbf{g}_{\mathbf{u}} \times\left(\mathbf{g}_{\mathrm{v}} \times \mathbf{g}_{\mathrm{w}}\right)=\mathbf{g}_{\mathbf{u}} \times \mathbf{g}_{\mathrm{v} \circ \mathrm{w}}=\mathbf{g}_{\mathbf{u} \circ(\mathrm{v} \circ \mathrm{w})}=\mathbf{g}_{(\mathbf{u} \circ \mathbf{v}) \circ \mathrm{w}}=\mathbf{g}_{\mathbf{u} \circ \mathbf{v}} \times \mathbf{g}_{\mathbf{w}}=\left(\mathbf{g}_{\mathbf{u}} \times \mathbf{g}_{\mathbf{v}}\right) \times \mathbf{g}_{\mathrm{w}}$.

Finally, the inverse element of $\mathbf{g}_{\mathbf{z}}$ is $\mathbf{g}_{\mathbf{z}^{-1}}$ since $\mathbf{g}_{\mathbf{z}} \times \mathbf{g}_{\mathbf{z}^{-1}}=\mathbf{g}_{\mathbf{z}^{\circ} \mathbf{z}^{-1}}=\mathbf{g}_{\mathbf{1}}$ for every $\mathbf{z} \in \overline{\mathbb{C}}^{n}$.

It is obvious that the mapping $\chi$ is bijective. Since $g_{z_{k}}\left(w_{k}\right)$ are analytic functions in $\overline{\mathbb{C}} \backslash\left\{a_{k}, 1 / a_{k}\right\}$, the function $\mathbf{g}_{\mathbf{z}}(\mathbf{w})$ is analytic in $\overline{\mathbb{C}}^{n} \backslash\left\{\mathbf{a}, \mathbf{a}^{-1}\right\}$. Obviously, $\mathbf{G}_{\mathbf{a}}$ as complex $n$-dimensional manifold has an analytic structure and then the isomorphism $\chi$ makes from $\mathbf{G}$ a Lie group with analytic structure as complex $n$-dimensional manifold.

We used [3] [4] [5] for the basic knowledge about Lie groups and their actions.

The actions by left and right translations of $\mathbf{G}$ on itself are defined as: $L\left(\mathbf{g}_{\mathbf{z}}, \mathbf{g}_{\mathbf{w}}\right)=\mathbf{g}_{\mathbf{z} \circ \mathbf{w}}$, respectively $R\left(\mathbf{g}_{\mathbf{z}}, \mathbf{g}_{\mathbf{w}}\right)=\mathbf{g}_{\mathbf{w} \circ \mathbf{z}^{-1}}$.

Theorem 1 implies that $\mathbf{G}$ acts freely and transitively on itself by left and right translations.

\section{Discrete Subgroups of G}

Let $\mathbf{z} \in \overline{\mathbb{C}}^{n}$ be an arbitrary element and for every $k \in \mathbb{Z}$ let us denote

$$
\mathbf{z}^{(k+1)}=\mathbf{z}^{(k)} \circ \mathbf{z}
$$

where $\mathbf{z}^{(0)}=\mathbf{1}$ and $\mathbf{z}^{(1)}=\mathbf{z}$.

Then, for every $j, k \in \mathbb{Z}$ we have $\mathbf{z}^{(j)} \circ \mathbf{z}^{(k)}=\mathbf{z}^{(j+k)}$ and using the formula (4), $\mathbf{g}_{\mathbf{z}^{(j)}} \times \mathbf{g}_{\mathbf{z}^{(k)}}=\mathbf{g}_{\mathbf{z}^{(j+k)}}$ for every $\mathbf{z} \in \overline{\mathbb{C}}^{n} \backslash\left\{\mathbf{a}, \mathbf{a}^{-1}\right\}$, in particular $\mathbf{g}_{\mathbf{z}^{(k)}} \times \mathbf{g}_{\mathbf{z}^{(-k)}}=\mathbf{g}_{z^{(0)}}=\mathbf{g}_{\mathbf{1}}$ which is the identity element in $\mathbf{G}$. It results that the group $\left\langle\mathbf{g}_{\mathbf{z}}\right\rangle$ generated by $\mathbf{g}_{\mathbf{z}}$ is a subgroup of $\mathbf{G}$. By Theorem 1 (2) we have that $\mathbf{z}^{(k+1)}=\mathbf{z}^{(k)}$ only if $\mathbf{z}=\mathbf{1}$, hence for every $k \neq 0$ we have $\mathbf{g}_{\mathbf{z}^{(k+1)}} \neq \mathbf{g}_{\mathbf{z}^{(k)}}$ and since for every $j \in \mathbb{Z}, \quad \mathbf{z} \neq \mathbf{a}$ implies $\mathbf{z}^{(j)} \neq \mathbf{a}$ then for $k \neq 0$ and $j \neq 0$, we have $\mathbf{g}_{\mathbf{z}^{(k+j)}} \neq \mathbf{g}_{\mathbf{z}^{(k)}}$, therefore the elements of $\left\langle\mathbf{g}_{\mathbf{z}}\right\rangle$ are all distinct.

Theorem 3. For every $\mathbf{z} \in \overline{\mathbb{C}}^{n} \backslash\left\{\mathbf{a}, \mathbf{a}^{-1}\right\}$, the group $\left\langle\mathbf{g}_{\mathbf{z}}\right\rangle$ generated by $\mathbf{g}_{\mathbf{z}}$ is a discrete subgroup of $\mathbf{G}$.

Proof. The case of $\mathbf{z}=\mathbf{1}$ is trivial. Suppose that for a given $\mathbf{z} \neq \mathbf{1}$ we would have $\lim _{k \rightarrow \infty} \mathbf{g}_{\mathbf{z}^{(k)}}=\mathbf{g} \in \mathbf{G}$ then $\mathbf{g}=\lim _{k \rightarrow \infty} \mathbf{g}_{\mathbf{z}^{(k+1)}}=\lim _{k \rightarrow \infty} \mathbf{g}_{\mathbf{z}^{(k)}} \times \mathbf{g}_{\mathbf{z}}=\mathbf{g} \times \mathbf{g}_{\mathbf{z}}$, which means that $\mathbf{g}_{\mathbf{z}}=\mathbf{g}_{1}$, contrary to the assumption.

Corollary 1. For every $\mathbf{z} \in \overline{\mathbb{C}}^{n} \backslash\{\mathbf{a}, \mathbf{1} / \mathbf{a}\}$ the subgroup $\left\langle\mathbf{g}_{\mathbf{z}}\right\rangle$ acts freely and properly discontinuously on $\mathbf{G}$ by left and by right translations.

\section{Antianalytic Involutions of $\overline{\mathbb{C}}^{n}$}

Let $n_{1}, n_{2}, \cdots, n_{k}$ be a non-empty subsequence of $1,2, \cdots, n$ and let

$$
h_{j}\left(z_{j}\right)=-1 / \bar{z}_{j}, j=n_{1}, n_{2}, \cdots, n_{k}, h_{j}\left(z_{j}\right)=z_{j}, j \neq n_{1}, n_{2}, \cdots, n_{k} .
$$

Then the mapping $\mathbf{h}: \overline{\mathbb{C}}^{n} \rightarrow \overline{\mathbb{C}}^{n}$ defined by 


$$
\mathbf{h}(\mathbf{z})=\left(h_{1}\left(z_{1}\right), h_{2}\left(z_{2}\right), \cdots, h_{n}\left(z_{n}\right)\right)
$$

is a fixed point free involution of $\overline{\mathbb{C}}^{n}$ in the sense that some of the mappings $h_{j}\left(z_{j}\right)$ are fixed point free involutions of $\overline{\mathbb{C}}$, while the others are the identity mapping. Then, it is true for $\mathbf{h}$ itself that for every $\mathbf{z} \in \overline{\mathbb{C}}^{n}$ we have $\mathbf{h}(\mathbf{h}(\mathbf{z}))=\mathbf{z}$. Moreover, there is no $\mathbf{z}=\left(z_{1}, z_{2}, \cdots, z_{n}\right) \in \overline{\mathbb{C}}^{n}$ for which $\mathbf{h}(\mathbf{z})=\mathbf{z}$, since this would imply $h_{j}\left(z_{j}\right)=z_{j}$ for every $j$ and if $j=n_{1}, n_{2}, \cdots, n_{k}$ this would mean $\left|z_{j}\right|^{2}=-1$, which is absurd. Since $h_{j}, j=n_{1}, n_{2}, \cdots, n_{k}$ are antianalytic self mappings of $\overline{\mathbb{C}}$, we will say that $\mathbf{h}$ is antianalytic.

Let us notice that the functions $\mathbf{h}$ of the form we just listed are not the only antianalytic involutions of $\overline{\mathbb{C}}^{n}$. If for $a \in \mathbb{C},|a|<1$ and $\alpha \in \mathbb{R}$ we take the Möbius transformation $M(z)=\mathrm{e}^{i \alpha} \frac{z-a}{1-\bar{a} z}$ which maps the unit disc onto itself, the unit circle onto itself and the exterior of the unit disc onto itself, we can prove:

Theorem 4. The function $M^{-1} \circ h \circ M(z)$, where $h(M(z))=-1 / \overline{M(z)}$, is a fixed point free antianalytic involution of $\overline{\mathbb{C}}$.

Proof. We have that

$$
\begin{gathered}
h(M(z))=-\mathrm{e}^{i \alpha} \frac{1-a \bar{z}}{\bar{z}-\bar{a}} \\
M^{-1}(w)=\frac{w+a \mathrm{e}^{i \alpha}}{\bar{a} w+\mathrm{e}^{i \alpha}}, \\
M^{-1}(h(M(z)))=\frac{h(M(z))+a \mathrm{e}^{i \alpha}}{\bar{a} h(M(z))+\mathrm{e}^{i \alpha}} \\
=\left[-\mathrm{e}^{i \alpha} \frac{1-a \bar{z}}{\bar{z}-\bar{a}}+a \mathrm{e}^{i \alpha}\right] /\left[-\bar{a} \mathrm{e}^{i \alpha} \frac{1-a \bar{z}}{z-\bar{a}}+\mathrm{e}^{i \alpha}\right] \\
\left.=\frac{2 a \bar{z}-\left(1+|a|^{2}\right)}{\left(1+|a|^{2}\right) \bar{z}-2 \bar{a}}\right] \\
\frac{2 \bar{a} z-\left(1+|a|^{2}\right)}{\left(1+|a|^{2}\right) z-2 a}
\end{gathered}
$$

which shows that $M^{-1} \circ h \circ M(z)$ is antianalytic, since its complex conjugate is analytic. The equality $M^{-1} \circ h \circ M(z)=z$ implies $h(M(z))=M(z)$, which is impossible since $h$ is fixed point free, hence $M^{-1} \circ h \circ M(z)$ is fixed point free. Finally,

$$
\begin{aligned}
{\left[M^{-1} \circ h \circ M(z)\right] \circ\left[M^{-1} \circ h \circ M(z)\right] } & =M^{-1} \circ h \circ M \circ M^{-1} \circ h \circ M(z) \\
& =M^{-1} \circ h \circ h \circ M(z) \\
& =M^{-1} \circ M(z)=z
\end{aligned}
$$

showing that $M^{-1} \circ h \circ M(z)$ is an involution.

We keep the notation $\mathbf{h}$ for any antianalytic involution of $\overline{\mathbb{C}}^{n}$ constructed with the functions of this type by the method of the previous paragraph.

A given antianalytic involution $\mathbf{h}$ and the identity mapping of $\overline{\mathbb{C}}^{n}$ form a 
group of transformations $\langle\mathbf{h}\rangle$ of $\overline{\mathbb{C}}^{n}$.

Theorem 5. The quotient space $\overline{\mathbb{C}}^{n} /\langle\mathbf{h}\rangle$ can be endowed with a differentiable manifold structure, so that it becomes a non orientable differentiable manifold.

Proof. Indeed, an analytic atlas can be created on $\overline{\mathbb{C}}^{n}$ such that the local chart for any point $\mathbf{z} \in \mathbb{C}^{n}$ is the identity on $\mathbb{C}^{n}$. Next, if for a $\mathbf{w}=\left(w_{1}, w_{2}, \cdots, w_{n}\right)$ we have $w_{j}=\infty$ when $j=n_{1}, n_{2}, \cdots, n_{k}$ local charts $(\mathbf{U}, \varphi)$ can be used, where $\mathbf{U}=\overline{\mathbb{C}}^{n} \backslash\{\boldsymbol{\zeta}\}$ with $\zeta_{j}=w_{j}$ for $j=n_{1}, n_{2}, \cdots, n_{k}$ and $\zeta_{j}=0$ otherwise and $\varphi_{j}\left(z_{j}\right)=z_{j}$ when $j=n_{1}, n_{2}, \cdots, n_{k}$ and $\varphi_{j}\left(z_{j}\right)=1 / z_{j}$ otherwise. Obviously, $\overline{\mathbb{C}}^{n}$ with such an atlas is a differentiable manifold for which every change of chart is a complex analytic function. The projection function $\pi: \overline{\mathbb{C}}^{n} \rightarrow \overline{\mathbb{C}}^{n} /\langle\mathbf{h}\rangle$, by which we have $\pi(\mathbf{z})=\pi(\mathbf{h}(\mathbf{z}))$ for every $\mathbf{z} \in \overline{\mathbb{C}}^{n}$, induces a differentiable manifold structure on $\overline{\mathbb{C}}^{n} /\langle\mathbf{h}\rangle$. Indeed, to every chart $(\mathbf{U}, \varphi)$ on $\overline{\mathbb{C}}^{n}$ corresponds a chart $(\mathbf{U}, \psi)$ on $\overline{\mathbb{C}}^{n} /\langle\mathbf{h}\rangle$, where $\psi=\varphi \circ \pi_{\mathbf{U}}^{-1}$ and if $\mathbf{U}_{2}=\mathbf{h}\left(\mathbf{U}_{1}\right)$ then $\pi_{\mathbf{U}_{1}} \circ \pi_{\mathbf{U}_{2}}^{-1}=\mathbf{h}$, hence $\psi_{2} \circ \psi_{1}^{-1}=\varphi_{2} \circ \pi_{\mathbf{U}_{2}}^{-1} \circ \pi_{\mathbf{U}_{1}} \circ \varphi_{1}^{-1}=\varphi_{2} \circ \mathbf{h} \circ \varphi_{1}^{-1}$. This structure is no more analytic since every change of charts $\varphi_{2} \circ \mathbf{h} \circ \varphi_{1}^{-1}$ is antianalytic. However, it is harmonic and therefore of class $C^{\infty}$.

Theorem 6. For any fixed point free antianalytic involution $\mathbf{h}$ of $\overline{\mathbb{C}}^{n}$ there is a partition of $\overline{\mathbb{C}}^{n}$ into two sets $\mathbf{H}$ and $\mathbf{K}$ such that $\mathbf{z} \in \mathbf{H}$ if and only if $\mathbf{h}(\mathbf{z}) \in \mathbf{K}$. With the induced topology of $\overline{\mathbb{C}}^{n}$ the topological spaces $\mathbf{H}$ and $\mathbf{K}$, as well as $\overline{\mathbb{C}}^{n} /\langle\mathbf{h}\rangle$ are homeomorphic under the projection $\pi(\mathbf{z})=\langle\mathbf{z}, \mathbf{h}(\mathbf{z})\rangle$.

Proof. We give a constructive proof. Let $\mathbf{z}_{1} \in \overline{\mathbb{C}}^{n}$ be arbitrary. Since $\mathbf{h}$ is a fixed point free involution of $\overline{\mathbb{C}}^{n}$ we have that $\mathbf{h}\left(\mathbf{z}_{1}\right) \neq \mathbf{z}_{1}$. Then there are disjoint open neighborhoods $\mathbf{U}_{1}$ of $\mathbf{z}_{1}$ and $\mathbf{V}_{1}$ of $\mathbf{h}\left(\mathbf{z}_{1}\right)$ such that $\mathbf{z} \in \mathbf{U}_{1}$ if and only if $\mathbf{h}(\mathbf{z}) \in \mathbf{V}_{1}$. Let $\mathbf{z}_{2} \in \overline{\mathbb{C}}^{n} \backslash\left(\mathbf{U}_{1} \cup \mathbf{V}_{1}\right)$ be arbitrary. We infer that $\mathbf{h}\left(\mathbf{z}_{2}\right) \in \overline{\mathbb{C}}^{n} \backslash\left(\mathbf{U}_{1} \cup \mathbf{V}_{1}\right)$. Indeed, supposing $\mathbf{h}\left(\mathbf{z}_{2}\right) \in \mathbf{U}_{1}$ would imply that $\mathbf{z}_{2}=\mathbf{h}\left(\mathbf{h}\left(\mathbf{z}_{2}\right)\right) \in \mathbf{V}_{1}$, contrary to the hypothesis. Analogously we find a contradiction supposing $\mathbf{h}\left(\mathbf{z}_{2}\right) \in \mathbf{V}_{1}$. Then there are open disjoint neighborhoods $\mathbf{U}_{2}$ of $\mathbf{z}_{2}$ and $\mathbf{V}_{2}$ of $\mathbf{h}\left(\mathbf{z}_{2}\right)$ such that $\mathbf{z} \in \mathbf{U}_{2}$ if and only if $\mathbf{h}(\mathbf{z}) \in \mathbf{V}_{2}$. Moreover, we can take $\mathbf{U}_{2}$ such that $\left(\mathbf{U}_{1} \cup \mathbf{U}_{2}\right) \cap\left(\mathbf{V}_{1} \cup \mathbf{V}_{2}\right)=\varnothing$. In this way we can build two sequences of open sets $\left(\mathbf{U}_{n}\right)$ and $\left(\mathbf{V}_{n}\right)$ such that $\mathbf{U}=\bigcup_{n=1}^{\infty} \mathbf{U}_{n}$ and $\mathbf{V}=\bigcup_{n=1}^{\infty} \mathbf{V}_{n}$ are disjoint and $\mathbf{z} \in \mathbf{U}$ if and only if $\mathbf{h}(\mathbf{z}) \in \mathbf{V}$. To make sure that the process ends in a countable number of steps, we can decide to take all the points $\mathbf{z}_{n}$ such that their coordinates in $\mathbb{R}^{2 n}$ are rational. Moreover, $\mathbf{U}$ and $\mathbf{V}$ are then maximal in the sense that $\mathbf{F}=\overline{\mathbb{C}}^{n} \backslash(\mathbf{U} \cup \mathbf{V})$ does not contain any open set. The set $\mathbf{F}$ with the trace atlas of $\overline{\mathbb{C}}^{n}$ is a complex differentiable manifold of dimension less than $n$. We repeat the process for $\mathbf{F}$ and we find that there are two relatively open maximal sets $\mathbf{U}^{\prime}$ and $\mathbf{V}^{\prime}$ such that $\mathbf{z} \in \mathbf{U}^{\prime}$ if and only if $\mathbf{h}(\mathbf{z}) \in \mathbf{V}^{\prime}$. Moreover, $\mathbf{F}^{\prime}=\mathbf{F} \backslash\left(\mathbf{U}^{\prime} \cup \mathbf{V}^{\prime}\right)$ is a complex differentiable manifold of dimension less than that of $\mathbf{F}$ and the process continues $k \leq n$ steps until we obtain dimension 0 . Then $\mathbf{F}^{(k)}$ is a countable set 
such that $\mathbf{z} \in \mathbf{F}^{(k)}$ if and only if $\mathbf{h}(\mathbf{z}) \in \mathbf{F}^{(k)}$. Then a partition of $\mathbf{F}^{(k)}$ into $\mathbf{U}^{(k)}$ and $\mathbf{V}^{(k)}$ such that $\mathbf{z} \in \mathbf{U}^{(k)}$ if and only if $\mathbf{h}(\mathbf{z}) \in \mathbf{V}^{(k)}$ is straightforward. Let us denote $\mathbf{H}=\mathbf{U} \cup \mathbf{U}^{\prime} \cup \cdots \cup \mathbf{U}^{(k)}$ and $\mathbf{K}=\mathbf{V} \cup \mathbf{V}^{\prime} \cup \cdots \cup \mathbf{V}^{(k)}$. Then $\mathbf{H}$ and $\mathbf{K}$ are such that $\mathbf{H} \cup \mathbf{K}=\overline{\mathbb{C}}^{n}, \mathbf{H} \cap \mathbf{K}=\varnothing$ and $\mathbf{z} \in \mathbf{H}$ if and only if $\mathbf{h}(\mathbf{z}) \in \mathbf{K}$.

It is obvious that $\pi_{\mid \mathrm{H}}$ and $\pi_{\mid \mathrm{K}}$ are one-to-one and onto functions and if the topology of $\overline{\mathbb{C}}^{n} /\langle\mathbf{h}\rangle$ is chosen such that the projection $\pi(\mathbf{z})$ is continuous, then the three topological spaces are homeomorphic.

We needed this construction for the following reason. The notation $\tilde{\mathbf{z}}=(\mathbf{z}, \mathbf{h}(\mathbf{z}))$ is ambiguous in the sense that on the right hand side we have an ordered couple of points, while in reality for the $\tilde{\mathbf{z}} \in \overline{\mathbb{C}}^{n} /\langle\mathbf{h}\rangle$ the order does not count and there is a situation which will appear later where this fact is essential. Now we can decide that once $\mathbf{H}$ and $\mathbf{K}$ have been built, they will remain permanently the same and every time we meet a couple $(\mathbf{z}, \mathbf{h}(\mathbf{z}))$ we have chosen $\mathbf{z} \in \mathbf{H}$. It is as if we ignore occasionally the existence of $\mathbf{K}$ and instead of $\overline{\mathbb{C}}^{n} /\langle\mathbf{h}\rangle$ we work only with $\mathbf{H}$.

We can define an operation on $\overline{\mathbb{C}}^{n} /\langle\mathbf{h}\rangle$ by using the composition law in $\overline{\mathbb{C}}^{n}$ from the section 1 . For every couple $\tilde{\mathbf{z}}$ and $\tilde{\mathbf{w}}$ from $\overline{\mathbb{C}}^{n} /\langle\mathbf{h}\rangle$ we write $\tilde{\mathbf{z}} \cdot \tilde{\mathbf{w}}=\widetilde{\mathbf{z} \circ \mathbf{w}}$, where $\mathbf{z}, \mathbf{w} \in \mathbf{H}$.

Theorem 7. The multiplication $\tilde{\mathbf{z}} \cdot \tilde{\mathbf{w}}$ is an internal composition law in $\overline{\mathbb{C}}^{n} /\langle\mathbf{h}\rangle$ with the unit element $\tilde{\mathbf{1}}=(\mathbf{1}, \mathbf{h}(\mathbf{1}))$ and $\widetilde{\mathbf{z}^{-1}}$, the inverse element of $\tilde{\mathbf{z}}$. The multiplication is commutative but not associative and therefore this law does not define a structure of Lie group on $\overline{\mathbb{C}}^{n} /\langle\mathbf{h}\rangle$.

Proof. It is obvious that for every $\mathbf{z}, \mathbf{w} \in \overline{\mathbb{C}}^{n} /\langle\mathbf{h}\rangle, \widetilde{\mathbf{z} \circ \mathbf{w}}$ is well defined and represents an element of $\overline{\mathbb{C}}^{n} /\langle\mathbf{h}\rangle$. Moreover, $\tilde{\mathbf{z}} \cdot \tilde{\mathbf{w}}=\widetilde{\mathbf{z} \circ \mathbf{W}}=\widetilde{\mathbf{W} \circ \mathbf{z}}=\tilde{\mathbf{w}} \cdot \tilde{\mathbf{z}}$, $\tilde{\mathbf{1}} \cdot \tilde{\mathbf{z}}=\widetilde{\mathbf{1} \circ \mathbf{z}}=\tilde{\mathbf{z}}$ and $\tilde{\mathbf{z}} \cdot \widetilde{\mathbf{z}^{-1}}=\widetilde{\mathbf{z} \circ \mathbf{z}^{-1}}=\tilde{\mathbf{1}}$. The non associativity of the law comes from the fact that although $\mathbf{z}, \mathbf{w} \in \mathbf{H}$ it may happen that $\mathbf{z} \circ \mathbf{w} \in \mathbf{K}$ and then the expression $\widetilde{\mathbf{Z} \circ \mathbf{W}} \cdot \tilde{\mathbf{z}}$ has no meaning.

We were expecting that one of the group axioms of the multiplication in $\overline{\mathbb{C}}^{n} /\langle\mathbf{h}\rangle$ not to be satisfied, since otherwise this manifold would be a Lie group and it is known (see [5], page 140) that every Lie group is an orientable manifold. However, as proven in the next theorem, actions of Lie groups on such a manifold exist.

Theorem 8. The mapping $\alpha: \mathbf{G} \times\left(\overline{\mathbb{C}}^{n} /\langle\mathbf{h}\rangle\right) \rightarrow \overline{\mathbb{C}}^{n} /\langle\mathbf{h}\rangle$ defined by $\alpha\left(\mathbf{g}_{\mathbf{z}}, \tilde{\mathbf{w}}\right)=\widetilde{\mathbf{z} \circ \mathbf{W}}$ is a left action of the Lie group $\mathbf{G}$ on the non orientable manifold $\overline{\mathbb{C}}^{n} /\langle\mathbf{h}\rangle$.

Proof. The mapping is obviously of class $C^{\infty}$. Moreover, $\alpha\left(\mathbf{g}_{1}, \tilde{\mathbf{w}}\right)=\widetilde{\mathbf{1} \circ \mathbf{W}}=\tilde{\mathbf{w}}$ for every $\tilde{\mathbf{w}} \in \overline{\mathbb{C}}^{n} /\langle\mathbf{h}\rangle$. Finally, $\alpha\left(\mathbf{g}_{\mathbf{z}}, \alpha\left(\mathbf{g}_{\zeta}, \tilde{\mathbf{w}}\right)\right)=\alpha\left(\mathbf{g}_{\mathbf{z}}, \overline{\boldsymbol{\zeta} \circ \mathbf{w}}\right)=\overline{\mathbf{z} \circ(\boldsymbol{\zeta} \circ \mathbf{w})}=\overline{(\mathbf{z} \circ \boldsymbol{\zeta}) \circ \mathbf{w}}=\alpha\left(\mathbf{g}_{\mathbf{z} \circ \zeta}, \tilde{\mathbf{w}}\right)=\alpha\left(\mathbf{g}_{\mathbf{z}} \times \mathbf{g}_{\zeta}, \tilde{\mathbf{w}}\right)$, hence indeed, $\alpha$ is a left action of the Lie group $\mathbf{G}$ on the non orientable manifold $\overline{\mathbb{C}}^{n} /\langle\mathbf{h}\rangle$.

Corollary 2. The mapping $\beta:\left(\overline{\mathbb{C}}^{n} /\langle\mathbf{h}\rangle\right) \times \mathbf{G} \rightarrow \overline{\mathbb{C}}^{n} /\langle\mathbf{h}\rangle$ defined by 
$\beta\left(\tilde{\mathbf{w}}, \mathbf{g}_{\mathbf{z}}\right)=\overline{\mathbf{w} \circ \mathbf{z}^{-1}}$ is a right action of $\mathbf{G}$ on $\overline{\mathbb{C}}^{n} /\langle\mathbf{h}\rangle$, since

$\beta\left(\tilde{\mathbf{w}}, \mathbf{g}_{\mathbf{z}}\right)=\alpha\left(\mathbf{g}_{\mathbf{z}}^{-1}, \tilde{\mathbf{w}}\right)$, as it can be easily checked.

By the general theory of Lie groups, these actions of $\mathbf{G}$ on $\overline{\mathbb{C}}^{n} /\langle\mathbf{h}\rangle$ define homomorphisms $t$ from the Lie group $\mathbf{G}$ to the group $\operatorname{Diff}\left(\overline{\mathbb{C}}^{n} /\langle\mathbf{h}\rangle\right)$ of diffeomorphisms of $\overline{\mathbb{C}}^{n} /\langle\mathbf{h}\rangle$ such that $t\left(\mathbf{g}_{\mathbf{z}}\right)$ is the mapping $\tilde{\mathbf{w}} \rightarrow \alpha\left(\mathbf{g}_{\mathbf{z}}, \tilde{\mathbf{w}}\right)$, respectively $\tilde{\mathbf{w}} \rightarrow \beta\left(\tilde{\mathbf{w}}, \mathbf{g}_{\mathbf{z}}\right)$. Reciprocally, every homomorphism $t: \mathbf{G} \rightarrow \operatorname{Diff}\left(\overline{\mathbb{C}}^{n} /\langle\mathbf{h}\rangle\right)$ defines left and right actions of $\mathbf{G}$ on $\overline{\mathbb{C}}^{n} /\langle\mathbf{h}\rangle$ by $\alpha(\mathbf{g}, \tilde{\mathbf{w}})=t(\mathbf{g}) \cdot \tilde{\mathbf{w}}$, respectively $\beta(\tilde{\mathbf{w}}, \mathbf{g})=t\left(\mathbf{g}^{-1}\right) \cdot \tilde{\mathbf{w}}$.

\section{Conclusion}

Non orientable $n$-dimensionl complex manifolds can be obtained by factorization with a two elements group generated by an antianalytic involution of $\overline{\mathbb{C}}^{n}$. Such involutions can be obtained, for example, composing in some coordinate planes Möbius transformations of the form $M\left(z_{k}\right)=\mathrm{e}^{i \theta_{k}}\left(z_{k}-a_{k}\right) /\left(1-\bar{a}_{k} z_{k}\right)$, $\left|a_{k}\right|<1$ with the mappings $h_{k}(z)=-1 / \bar{z}_{k}$. An internal composition law can be defined on such a manifold with the help of some bi-Möbius transformations and actions of Lie groups on the respective manifold can be put into evidence. We realized this task by devising an appropriate partition of that manifold.

\section{Conflicts of Interest}

The authors declare no conflicts of interest regarding the publication of this paper.

\section{References}

[1] Barza, I. and Ghisa, D. (1997) Lie Groups Actions on the Möbius Strip, Topics in Complex Analysis. In: Dimiev, S. and Sekigawa, K., Eds., Differential Geometry and Physics, World Sci., Singapore, 62-72.

[2] Barza, I. and Ghisa, D. (2020) Lie Groups Actions on Non Orientable Klein Surfaces. In: Dobrev V., Ed., Lie Theory and Its Applications in Physics, Springer, Singapore, Vol. 335, 421-428. https://doi.org/10.1007/978-981-15-7775-8_33

[3] Boothby, W.M. (1986) An Introduction to Differential Manifolds and Riemannian Geometry. Academic Press Inc., Cambridge.

[4] Warner, F.W. (1983) Foundations of Differential Geometry and Lie Groups. In: Graduate Texts in Mathematics, Springer-Verlag, New York, Vol. 94.

https://doi.org/10.1007/978-1-4757-1799-0

[5] Onishchik, A.L. (Editor) (1993) Lie Groups and Lie Algebras I. In: Encyclopaedia of Mathematical Sciences, Springer-Verlag, Berlin, Vol. 20. https://doi.org/10.1007/978-3-642-57999-8 